\title{
INFLUÊNCIA DE POLIACRILAMIDA NA REDUÇÃO DA PERDA DE CARGA EM TUBULAÇÃO DE POLIETILENO
}

\author{
André Luiz Justi ${ }^{1}$, João Luis Zocoler ${ }^{2}$, Lucas da Costa Santos ${ }^{3}$
}

\section{RESUMO}

Analisar a perda de carga nas tubulações é de suma importância para projetar de forma adequada sistemas de irrigação, pois a mesma influência no sistema de bombeamento, afetando o custo do projeto e também a demanda energética do mesmo. O presente trabalho objetivou analisar a influência do uso do polímero poliacrilamida como agente redutor do atrito hidrodinâmico em tubos de polietileno e verificar a redução da perda de carga nos mesmos. O estudo foi realizado no Laboratório de Ensaio de Equipamentos para Irrigação do Departamento de Engenharia Rural pertencente à Faculdade de Ciências Agronômica de Botucatu - SP, utilizando três diâmetros de tubos, 1, 3/4 e 1/2 polegada, com cinco vazões, $0352,0,225,0,125,0,05$ e $0,0175 \mathrm{~L} \mathrm{~s}^{-1}$, buscando simular os regimes laminar, transição e turbulento de escoamento, com concentração de $10 \mathrm{mg} \mathrm{L}^{-1}$ de polímero. Os resultados obtidos foram positivos quanto a redução na perda de carga, com valores que chegaram até 76,93\%, utilizando o tubo de 1 polegada, e também significativas reduções no fator de atrito. Diante dos resultados obtidos, pode-se concluir que o uso de poliacrilamida é viável em relação à quantidade necessária para obter resultados satisfatórios na redução na perda de carga.

Palavras-chave: atrito hidrodinâmico, bombeamento, irrigação.

\section{ABSTRACT}

\section{INFLUENCE OF POLYACRYLAMIDE ON HEAD LOSS REDUCTION IN POLYETHYLENE PIPES}

Analysis of head loss in pipes is important for effective designing of irrigation systems, because of its influence on pumping system, project cost and energy demand. This study was done to determine the possibility of using polyacrylamide polymer as hydrodynamic friction reducing agent in polyethylene pipes to reduce head loss. The study was conducted at Irrigation Equipment Testing Laboratory of the Rural Engineering Department belonging to Faculty of Agronomic Sciences, Botucatu - SP. The tests were conducted using pipe diameter of 1, 3/4 or 1/2 inch, at the flow rates of $0.352,0.225,0.125,0.05$ or $0.0175 \mathrm{~L} \mathrm{~s}^{-1}$, to simulate the laminar, transition and turbulent flow with the polymer concentration of $10 \mathrm{mg} \mathrm{L}^{-1}$. The head loss reduction reached $76.93 \%$ in one inch pipes. The reduction of friction factor was also significant. The data showed that the use of polyacrylamide is feasible considering quantity necessary to obtain satisfactory results.

Keywords: hydrodynamic friction, pumping, irrigation.

\section{Recebido para publicação em 06/05/2012. Aprovado em 03/10/2012.}

1- Engenheiro Agrícola, Doutorando em Agronomia - Irrigação e Drenagem, Unesp/Botucatu - SP, Professor Departamento de Solos e Engenharia Agrícola - Universidade Federal do Paraná - UFPR, aljusti@fca.unesp.br 2- Engenheiro Agrônomo, Professor Departamento de Fitossanidade Engenharia Rural e Solos, Unesp/Ilha Solteira -SP, zocoler@agr.feis.unesp.br

3- Engenheiro Agrônomo, Doutorando em Engenharia de Sistemas Agrícolas, ESALQ/USP - Piracicaba-SP, lucas.cs21@gmail.com

\section{REVENG} $460-466 \mathrm{p}$. 


\section{INTRODUÇÃO}

O sucesso de implantação da agricultura irrigada depende da otimização do projetohidráulico, buscando sempre a menor soma dos custos fixos e variáveis. Neste caso, um dos parâmetros mais importantes é a perda de carga, a qual deve ser determinada com precisão, resultando no sistema de recalque mais econômico (ANDRADE \& CARVALHO, 2001).

O cálculo de perdas de carga em situações que envolvam fluxo de água em tubulações é fonte constante de estudos, uma vez que esse fator referese à perda de energia provocada por atritos que ocorrem entre a água e as paredes das tubulações, como consequência da interação entre viscosidade e rugosidade, sendo refletida nos custos variáveis da instalação (KAMAND, 1988) e, conforme Bombardelli e Garcia (2003), assumindo a rugosidade constante, o valor da perda de carga calculada pelas equações empíricas pode variar significativamente quando comparado com o calculado pela equação de Darcy-Weisbach, onde o fator de atrito varia conforme as condições de escoamento.

$\mathrm{O}$ tipo de fluido e, consequentemente, a sua perda de carga em tubulações, são fundamentais no dimensionamento das tubulações e das bombas desses sistemas, uma vez que a altura manométrica representa a energia fornecida ao líquido suficiente para vencer o desnível geométrico e as perdas de carga ocorridas ao longo do percurso (CARVALHO et al., 2008).

Polímeros, surfatantes, suspensão de partículas insolúveis, tais como grãos finos ou fibras, e polímeros misturados com sabões ou fibras, são alguns tipos de agentes que produzem reduçãode atrito hidrodinâmico (RAH) (MORGAN \& McCORMICK, 1990). Alguns polímeros naturais (como goma guar e xantana) também produzem RAH, porém, normalmente requerem maior concentração para ter o mesmo efeito dos polímeros sintéticos (SELLIN et al.,1982).

De acordo com Goodwin e Hughes (2000), em tubos, a turbulência é atingida para valores do Número de Reynolds (Re) maiores do que cerca de 2300. A partir deste valor, as substâncias redutoras de atrito começam a atuar, conforme apresentado na Figura 1. O diagrama esquemático indica o comportamento hidrodinâmico idealizado de um determinado fluido puro (como, por exemplo, a água) e, comparativamente, os efeitos da presença do redutor de atrito hidrodinâmico (BIZOTTO et al, 2011).

Uma das aplicações mais significativas da redução de atrito hidrodinâmico está relacionada com o bombeamento de petróleo ao longo do oleoduto que corta o Alasca, ligando as Baias de Prudhoe a Valdez, por uma extensão de $1287 \mathrm{~km}$. Cerca de $30 \%$ de redução de atrito é atingida; porém, a cada $100 \mathrm{~km}$ se faz necessária a reaplicação do redutor de atrito devido à perda de eficácia ocorrida pela degradação das macromoléculas (KULICKE et al., 1989).

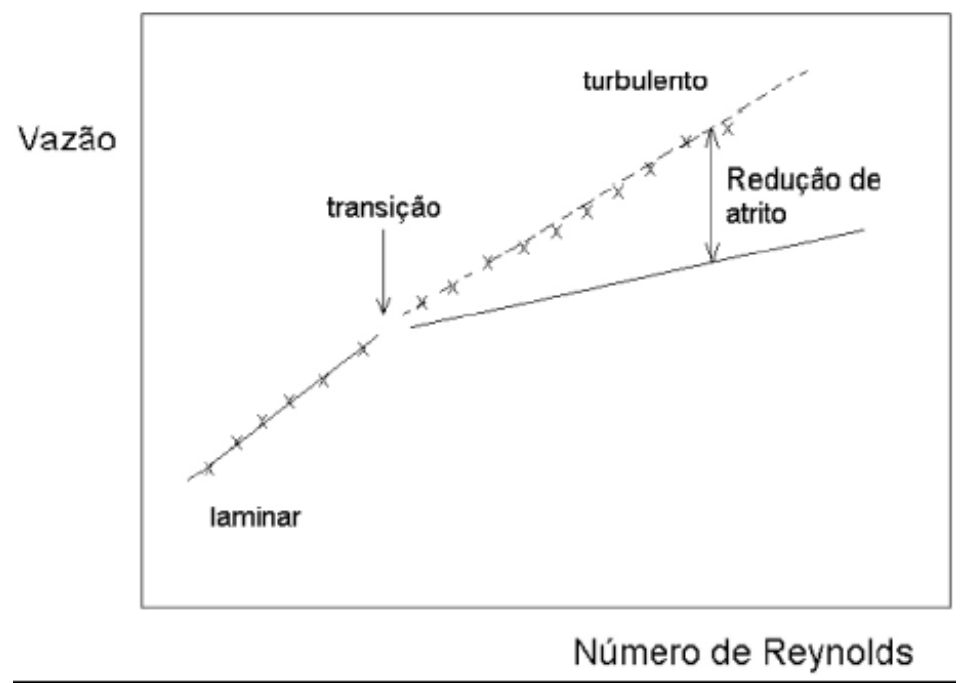

Figura 1. Diagrama característico representando a variação da vazão de um líquido puro (linha contínua) e contendo um aditivo redutor de atrito (x), em função do número de Reynolds. Adaptado de BIZOTTO et. al, 2011. 
Os polímeros mais estudados em sistemas aquosos são poli (óxido de etileno) (PEO) e poliacrilamida (PAM) (BAILEY \& KOLESKE, 1976). Ainda segundo esses autores, índices de até $80 \%$ de redução de atrito podem ser atingidos para estes sistemas em determinadas condições de escoamento.

Objetivou-se com este trabalho analisar a eficácia na redução da perda de carga em tubulação de polietileno usando o polímero poliacrilamida como agente redutor de atrito hidrodinâmico.

\section{MATERIAL E MÉTODOS}

O experimento foi conduzido no Laboratório de Ensaio de Equipamentos para Irrigação do Departamento de Engenharia Rural pertencente à Faculdade de Ciências Agronômica de Botucatu - SP.

Foi montado um sistema de forma que as tubulações pudessem ser acopladas junto a uma bomba centrífuga, marca KSB, modelo 32-16 $\mathrm{MB}$, ligada a uma chave de partida, com vazão máxima de $5 \mathrm{~m}^{3} \mathrm{~s}^{-1} \mathrm{e}$ altura manométrica de 32 m.c.a, abastecida por um reservatório para água com capacidade de $200 \mathrm{~L}$, sendo a tubulação de sucção com diâmetro de 2 polegadas, filtro de tela de 1 polegada, acoplado a um registro de esfera de 1 polegada, conforme mostra-se na Figura 2.

Para controle da vazão no sistema, foi utilizado um medidor de vazão marca SIGNET, modelo 8550-1, como mostra a Figura 2, alimentado por uma fonte de $12 \mathrm{~V}$, com exatidão de $0,5 \%$ da leitura a $25{ }^{\circ} \mathrm{C}$. De acordo com o manual do fabricante, foi feita a calibração do mesmo para realização do experimento. Antes do medidor de vazão, foi instalado um registro de esfera para tubulação de 1 polegada.

Para realizar o monitoramento da pressão durante os ensaios utilizou-se um manômetro digital com faixa de operação de 0 a $500 \mathrm{kPa}$. Para a leitura da perda de carga no sistema, foi utilizado um manômetro diferencial de mercúrio, com escala de leitura em milímetros.

Foram utilizados três configurações para o sistema montado, todas fazendo uso de tubulação de polietileno, uma com diâmetro nominal de 1 polegada e diâmetro interno de $22,96 \mathrm{~mm}$, uma com tubulação de $3 / 4$ de polegada de diâmetro e $18,20 \mathrm{~mm}$ de diâmetro interno e, por fim, uma configuração com tubo de diâmetro nominal de $1 / 2$ polegada e diâmetro interno de 12,09 $\mathrm{mm}$. Foram utilizadas cinco vazões diferentes para cada diâmetro ensaiado. Dessas vazões, três $\left(0352,0,225,0,125 \mathrm{~L} \mathrm{~s}^{-1}\right)$ para simular regime turbulento de escoamento, uma vazão $\left(0,05 \mathrm{~L} \mathrm{~s}^{-1}\right.$ para os tubos de 1 polegada e $3 / 4$ de polegada e $0,0375 \mathrm{~L} \mathrm{~s}^{-1}$ para o tubo de $1 / 2$ polegada) simulando regime de transição e para todos os diâmetros utilizados, uma vazão $\left(0,0175 \mathrm{~L} \mathrm{~s}^{-1}\right)$ para regime laminar de escoamento dos líquidos na tubulação, onde, na Quadro 1, são apresentados os valores médios

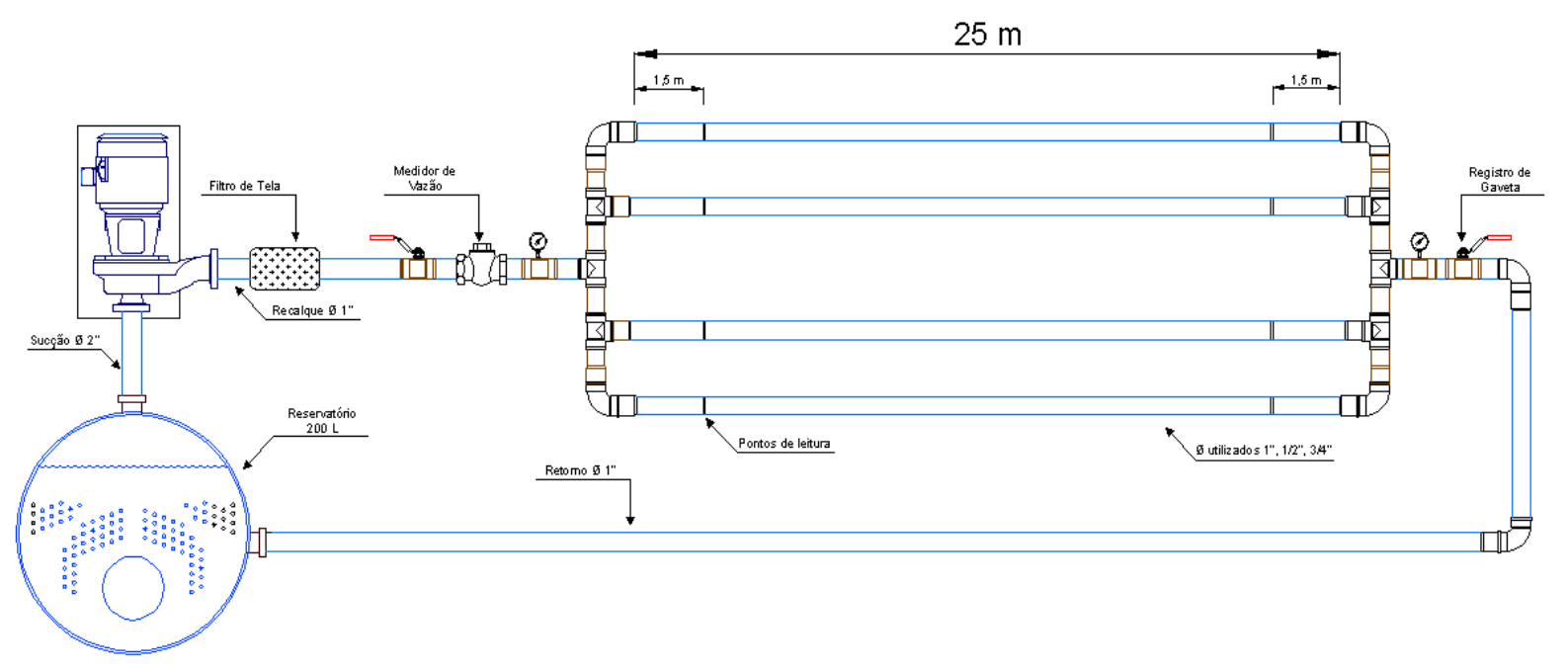

Figura 2. Layout do circuito montado para os ensaios. 
calculados do Número de Reynolds para cada vazão e diâmetro.

Para tomada de pressão, e verificar a perda de carga, foram instalados a uma distância de 1,5 metros do início da tubulação de polietileno, em cada tubo, um scalp intravenoso modelo borboleta, com agulha de $0,02 \mathrm{~m}$ de comprimento e $0,0008 \mathrm{~m}$ de diâmetro interno, com tubo de vinil transparente de $0,3 \mathrm{~m}$ de comprimento, como mostra a Figura 3, bem como instalados no fim da linha estudada, também a uma distância de 1,5 metros, resultando em um comprimento real de 22 metros de tubo para serem analisados. A temperatura média no período de realização dos ensaios foi de $15{ }^{\circ} \mathrm{C}$, sendo a viscosidade para essa temperatura de $1,146 \times 10^{-6} \mathrm{~m}^{2} \mathrm{~s}^{-1}$.

Foram realizados os ensaios com água $\mathrm{e}$ posteriormente, água com polímero redutor de atrito hidrodinâmico Poliacrilamida, na concentração de $10 \mathrm{mg} \mathrm{L}^{-1}$, sendo essa concentração indicada devido a natureza do tubo, pois, em concentrações maiores, o polímero poderia perder sua funcionalidade como redutor de atrito. O polímero foi diluído em uma solução estoque e posteriormente lançado no reservatório. As amostras de polímero foram fornecidas pela empresa SNF do Brasil Ltda, com nome comercial de FLONEX 9051 SI.

Quadro 1. Número de Reynolds utilizados para determinação dos regimes de escoamento

\begin{tabular}{|c|c|c|}
\hline $\begin{array}{l}\text { Vazão } \\
\left(\mathrm{L} \mathrm{s}^{-1}\right)\end{array}$ & $\begin{array}{c}\text { Diâmetro } \\
\text { (polegadas) }\end{array}$ & Número de Reynolds \\
\hline \multirow{3}{*}{0,352} & 1 & 15917,77 \\
\hline & $3 / 4$ & 18746,00 \\
\hline & $1 / 2$ & 26115,35 \\
\hline \multirow{3}{*}{0,225} & 1 & 11087,30 \\
\hline & $3 / 4$ & 13384,00 \\
\hline & $1 / 2$ & 17347,41 \\
\hline \multirow{3}{*}{0,125} & 1 & 6061,67 \\
\hline & $3 / 4$ & 8854,00 \\
\hline & $1 / 2$ & 10721,27 \\
\hline \multirow{3}{*}{$\mathbf{0 , 0 5 0}$} & 1 & 2853,68 \\
\hline & $3 / 4$ & 3408,00 \\
\hline & $1 / 2$ & 3497,14 \\
\hline \multirow{3}{*}{0,0175} & 1 & 964,19 \\
\hline & $3 / 4$ & 1410,00 \\
\hline & $1 / 2$ & 1750,22 \\
\hline
\end{tabular}
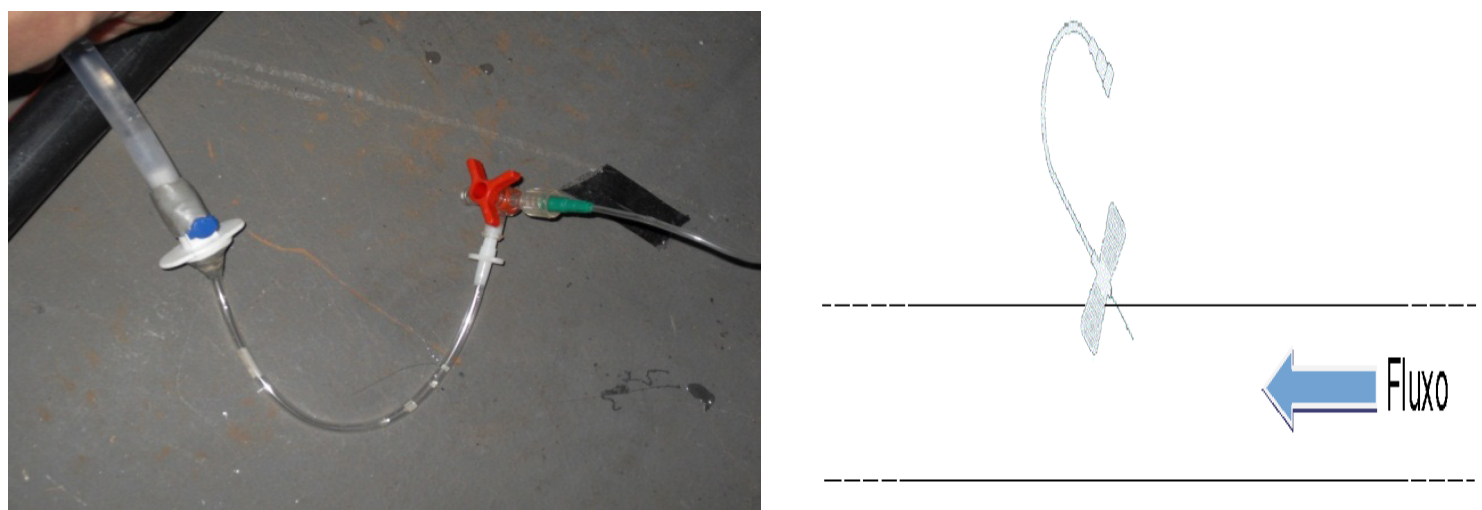

Figura 3. (a) Adaptação para uso dos Scalpis na tubulação; (b) Posição do Scalpi depois de fixo no tubo. 


\section{RESULTADOS E DISCUSSÃO}

No Quadro 2 apresentam-se os resultados obtidos de perda de carga ao longo do comprimento da tubulação, comparando o líquido com e sem polímero em função da variação do diâmetro, com a média de $0,2553 \mathrm{~m}$ e $0,1953 \mathrm{~m}$, para o diâmetro de 1 polegada, sem e com o uso do polímero, respectivamente. O diâmetro de $3 / 4$ de polegada apresentou média de perda de carga sem polímero de $0,6378 \mathrm{~m}$ e com polímero $0,4788 \mathrm{~m}$. Para o diâmetro de $1 / 2$ de polegada, a média sem polímero foi $2,0908 \mathrm{~m}$ e com polímero $1,8805 \mathrm{~m}$. Em relação às vazões, a comparação da perda de carga com a variação de vazão, os resultados são apresentados no Quadro 3, onde, na vazão de 0,05 $\mathrm{L} \mathrm{s}^{-1}$, foi encontrada uma perda de carga média de 0,0747 m sem polímero e 0,0294 m com polímero, com a vazão de $0,125 \mathrm{~L} \mathrm{~s}^{-1}$, sem polímero a média foi $0,5512 \mathrm{~m}$ e com polímero $0,4042 \mathrm{~m}$. A vazão de $0,225 \mathrm{~L} \mathrm{~s}^{-1}$ apresentou média sem polímero de $1,1980 \mathrm{~m}$ e com polímero $1,0489 \mathrm{~m}$. A maior vazão estudada, $0,352 \mathrm{~L} \mathrm{~s}^{-1}$, apresentou média de 2,1546 $\mathrm{m}$ sem polímero e com a adição do polímero a média de perda de carga encontrada foi $1,9236 \mathrm{~m}$.

Para a menor vazão, $0,0175 \mathrm{~L} \mathrm{~s}^{-1}$, que representava o regime laminar de escoamento, não foi detectada perda de carga no manômetro, fato associado ao comprimento dos tubos, o próprio regime de escoamento e líquido manométrico utilizado.

Shenoy (1984) afirmou que uma característica do fenômeno de redução de atrito hidrodinâmico em relação ao agente polimérico, é a ínfima quantidade de polímero requerida, da ordem de partes por milhão, de polímeros (geralmente

Quadro 2. Valores de perda de carga observados entre Líquido x Diâmetro

\begin{tabular}{ccc}
\hline Líquido & $\begin{array}{c}\text { Diâmetro } \\
\text { (polegada) }\end{array}$ & Perda de Carga (m) \\
\hline \multirow{2}{*}{ Sem polímero } & 1 & 0,2553 \\
& $3 / 4$ & 0,6378 \\
& $1 / 2$ & 2,0908 \\
Com polímero & 1 & 0,1953 \\
& $3 / 4$ & 0,4788 \\
\hline
\end{tabular}

Quadro 3. Valores de perda de carga observados entre Líquido x Vazão

\begin{tabular}{ccc}
\hline Vazão $\left(\mathbf{L ~ s}^{-1}\right)$ & Líquido & Perda de Carga $(\mathbf{m})$ \\
\hline \multirow{2}{*}{0,05} & Água sem polímero & 0,0747 \\
& Água com polímero & 0,0294 \\
0,125 & Água sem polímero & 0,5512 \\
& Água com polímero & 0,4042 \\
0,225 & Água sem polímero & 1,1980 \\
& Água com polímero & 1,0489 \\
& Água sem polímero & 2,1546 \\
\hline
\end{tabular}


lineares) e de elevada massa molar média (da ordem de $106 \mathrm{~g} \mathrm{~mol}^{-1}$ ), fato que se confirmou neste estudo, pois usou-se uma concentração de $10 \mathrm{mg}$ $\mathrm{L}^{-1}$ (aproximadamente $3 \mathrm{~g}$ ) para uma quantidade de água de 160 litros de água.

As diferenças observadas, confirmam o que Bizotto el al. (2011) afirmaram sobre o efeito TOM's, que macromoléculas de polímeros podem diminuir o atrito hidrodinâmico em líquidos.

Os valores de vazão também foram alterados, pois o sistema em sua máxima operação sem polímero trabalhou com uma vazão de $0,352 \mathrm{~L} \mathrm{~s}^{-1}$, sendo que após a aplicação do polímero, essa vazão passou para $0,572 \mathrm{~L} \mathrm{~s}^{-1}$ no tubo de 1 polegada, $0,545 \mathrm{~L} \mathrm{~s}^{-1} \mathrm{com} 3 / 4$ de polegada e $0,58 \mathrm{~L} \mathrm{~s}^{-1} \mathrm{com}$ o tubo de $1 / 2$ polegada, confirmando o que Bizotto et al. (2011) afirmaram, que uma boa redução de energia elétrica pode ser obtida usando-se bombas operando com menor potência para manter a mesma vazão ou, alternativamente, maior vazão pode ser obtida com as mesmas bombas operando na potencia nominal.

Warholic et al. (1999) usaram uma solução de poliacrilamida e acrilato de sódio, na água, encontrando uma redução significativa com uma concentração de $0,25 \mathrm{mg} \mathrm{L}^{-1}$. Afirmaram também que as máximas reduções foram observadas com concentrações entre 13 e $50 \mathrm{mg} \mathrm{L}^{-1}$, sendo que neste estudo, com o uso de uma concentração de $10 \mathrm{mg} \mathrm{L}^{-1}$ foi encontrada uma redução de atrito significativa, que gerou consideráveis valores de perda de carga nos tubos.

Os resultados obtidos neste estudo também se assemelham aos de Al-Yaari et al. (2009) em seu estudo conduzindo uma solução de água com polímero em tubos horizontais de 1 polegada, encontrando reduções de até $65 \%$ com concentrações entre 10 e $15 \mathrm{mg} \mathrm{L}^{-1}$.

\section{CONCLUSÕES}

Com base nos resultados obtidos, pode-se concluir que:

- O uso do polímero apresentou resultados positivos quanto à redução da perda de carga nos diâmetros e vazões estudados;
- Pode-se considerar viável sua utilização em função do custo relativamente baixo de aquisição; e

- Obteve-se vazões maiores com seu uso sem alterações no sistema, o que pode significar uma redução no custo com energia elétrica no bombeamento de água em sistemas de irrigação, ou ainda, redução na potência instalada nos sistemas de acionamento hidráulico.

\section{AGRADECIMENTOS}

À Coordenação de Aperfeiçoamento de Pessoal de Nível Superior Bolsista CAPES. Processo PSDE n $2378-11-6$.

\section{REFERÊNCIAS BIBLIOGRÁFICAS}

AL-YAARI, M., SOLEIMANI, A., ABUSHARKH, B., AL-MUBAIYEDH, U., ALSARKHI, A. Effect of drag reducing polymers on oil-water flow in a horizontal pipe. International Journal of Multiphase Flow, Baltimore, v.35, n.6, p.516-524, 2009.

ANDRADE, L., CARVALHO, J.A. Análise da equação de Swamee-Jain para cálculo do fator de atrito. Revista Brasileira de Engenharia Agrícola e Ambiental, Campina Grande, v.5, n.3, p.554-557, 2001.

BAILEY, F.E.; KOLESKE, V.J. Poly(ethylene oxide). Ed. Academic Press: New York, 1976, 173 p.

BIZOTTO, V.C.; ALKSCHBIRS, M.I.; SABADINI, E. Uma revisão sobre o efeito Toms - o fenômeno onde macromoléculas atenuam a turbulência em um líquido. Química Nova, São Paulo, v.34, n.4, 2011, p.658-664.

BOMBARDELLI, F.A.; GARCÍA, H. Hydraulic design of large-diameter pipes. Journal of Hydraulics Engineering. New York, v.129, n.11, 2003, p.839-846.

CARVALHO, J.A., AQUINO, R.F., PEREIRA, G.M.,RODRIGUES, R.C.,CAMPOS. Desempenho de bomba centrífuga operando com água residuária 
do processamento do café. Engenharia Agrícola. Jaboticabal, v.28, n.1, p.86-94, 2008.

GOODWIN, J.W.; HUGHES, R.W. Rheology for chemists-An introduction. The Royal Society of Chemistry: Cambridge, 2000, 290p.

KAMAND, F.Z. Hidraulic Friction Factors for Pipe Flow. Journal of Irrigation and Drainage Engineering, New York, v.114, n.2, p.311-323, 1988.

KULICKE, W.M.; ANDREIS, M.; GRAGER, H.; KOENING, J.L.; KOTER, M.; Advances in Polymer Science, Polymer Characterization/Polymer Solutions, Springer-Verlag: Berlin, 1989, 173p.

MORGAN, S.E.; McCORMICK, C.L.; WaterSoluble copolymers XXXII: Macromolecular dragreduction. A review of predictive theories and the effects of Polymer structure. Progress in Polymer Science, Pittsburgh, v.15, n.3, p.507-549, 1990.

SELLIN, R.H.J.; HOYT, J.W.; SCRIVENER, O. The effect of drag-reducing additives on fluid flows and their industrial applications. Part 1. Basic aspects. Journal of Hydraulic Research, Urbana, n.20, p.29-68, 1982.

SHENOY, A.V.A review on drag reduction with special reference to micellar systems. Colloid \& Polymer Science, Leipzig, v.262, n.4, p.319$337,1984$.

WARHOLIC, M.D; MASSAH, H.; HANRATTY, T.J. Influence of drag-reducing polymers on turbulence: Effects of Reynolds number, concentration and mixing. Experiments in Fluids, Heidelberg, v.27, n.5, p.461-472, 1999. 